EPJ Web of Conferences 66, 03093 (2014)

DOI: $10.1051 /$ epjconf/ 20146603093

(C) Owned by the authors, published by EDP Sciences, 2014

\title{
First EXL experiment with stored radioactive beam: Proton scattering on ${ }^{56} \mathrm{Ni}$
}

M. von Schmid ${ }^{1}$,a , S. Bagchi ${ }^{2}$, S. Bönig ${ }^{1}$, M. Csatlós ${ }^{3}$, I. Dillmann ${ }^{4}$, C. Dimopoulou ${ }^{4}$, P. Egelhof ${ }^{4}$, V. Eremin ${ }^{5}$, T. Furuno ${ }^{6}$, H. Geissel ${ }^{4}$, R. Gernhäuser ${ }^{7}$, M. N. Harakeh², A.-L. Hartig ${ }^{1}$, S. Ilieva ${ }^{1}$, N. Kalantar-Nayestanaki ${ }^{2}$, O. Kiselev ${ }^{4}$, H. Kollmus ${ }^{4}$, C. Kozhuharov ${ }^{4}$ A. Krasznahorkay ${ }^{3}$, T. Kröll ${ }^{1}$, M. Kuilman², S. Litvinov ${ }^{4}$, Yu. A. Litvinov ${ }^{4}$, M. Mahjour-Shafiei ${ }^{2,8}$, M. Mutterer ${ }^{4}$, D. Nagae ${ }^{9}$, M. A. Najafi ${ }^{2}$, C. Nociforo ${ }^{4}$, F. Nolden ${ }^{4}$, U. Popp ${ }^{4}$, C. Rigollet ${ }^{2}$, S. Roy ${ }^{2}$, C. Scheidenberger ${ }^{4}$, M. Steck ${ }^{4}$, B. Streicher ${ }^{2,4}$, L. Stuhl ${ }^{3}$, M. Thürauf ${ }^{1}$, T. Uesaka ${ }^{10}$, H. Weick ${ }^{4}$, J. S. Winfield ${ }^{4}$, D. Winters ${ }^{4}$, P. J. Woods ${ }^{11}$, T. Yamaguchi ${ }^{12}$, K. Yue ${ }^{1,4,13}$, J. C. Zamora ${ }^{1}$, and J. Zenihiro ${ }^{10}$

\footnotetext{
${ }^{1}$ Institut für Kernphysik, Technische Universität Darmstadt, 64289 Darmstadt, Germany

${ }^{2}$ Kernfysisch Versneller Instituut (KVI), Rijksuniversiteit Groningen, 9747 AA Groningen, Netherlands

${ }^{3}$ ATOMKI, Institute of Nuclear Research, Hungarian Academy of Sciences, 4026 Debrecen, Hungary

${ }^{4}$ GSI Helmholtzzentrum für Schwerionenforschung GmbH, 64291 Darmstadt, Germany

${ }^{5}$ loffe Physico-Technical Institute, Russian Academy of Sciences, St. Petersburg, 194021 Russia

${ }^{6}$ Division of Physics and Astronomy, Kyoto University, Kitashirakawa-Oiwakecho, Sakyo-ku, Kyoto 606-8502, Japan

${ }^{7}$ Physik-Department E12, Technische Universität München, 85748 Garching, Germany

${ }^{8}$ Department of Physics, University of Tehran, P.O. Box 14395/547, Tehran, Iran

${ }^{9}$ Department of Physics, University of Tsukuba, Tsukuba, Ibaraki 305-8577, Japan

${ }^{10}$ RIKEN Nishina Center, Wako, Saitama 351-0198, Japan

${ }^{11}$ Institute for Particle and Nuclear Physics, University of Edinburgh, Edinburgh, EH9 3JZ, United Kingdom

${ }^{12}$ Department of Physics, Saitama University, Saitama, Saitama 338-8570, Japan

${ }^{13}$ Institute of Modern Physics, Chinese Academy of Sciences, 730000 Lanzhou, China
}

\begin{abstract}
EXL (EXotic nuclei studied in Light-ion induced reactions at the NESR storage ring) is a project within NUSTAR at FAIR. It aims at investigations of light-ion induced direct reactions in inverse kinematics with radioactive ions cooled and stored in the future NESR (New Experimental Storage Ring). The existing ESR (Experimental Storage Ring) at GSI, together with its internal gas-jet target, provides a unique opportunity to perform such kind of experiments on a smaller scale at the present time. We have developed a UHV-compatible (Ultra High Vacuum) detector setup mainly based on DSSDs (Double-sided Silicon-Strip Detector) for the target-like recoils [4]. With this setup we were able to successfully investigate reactions with a stored radioactive beam for the first time ever. As a part of the first EXL campaign we investigated the reaction ${ }^{56} \mathrm{Ni}(\mathrm{p}, \mathrm{p})^{56} \mathrm{Ni}$ in inverse kinematics in order to measure the differential cross section for elastic proton scattering and deduce the nuclear-matter distribution and radius of ${ }^{56} \mathrm{Ni}$. In the following, the experimental setup is introduced in more detail, and the current status of the ongoing analysis is discussed.
\end{abstract}

\footnotetext{
ae-mail: schmid@ikp.tu-darmstadt.de
} 


\section{Introduction}

EXL is a project within NUSTAR [1] at FAIR [2]. Its aim is the study of nuclear structure of exotic nuclei with direct reactions in inverse kinematics. One of the key interests of EXL is the investigation of reactions at very low momentum transfer where, for example, the nuclear matter distribution, giant monopole resonances or Gamow-Teller transitions can be studied [3]. Performing these kind of experiments in storage rings leads to several advantages over conventional fixed target experiments. When aiming at more and more exotic nuclei, usually their lifetimes get shorter and the production rate of a radioactive beam drops. Both will limit the feasibility of experiments and the study of those nuclei. If the lifetimes allow to store the beam in a ring, the low rates can be compensated by the high revolution frequency of the stored ions in the ring, which is in the order of a few MHz. Hence, this allows for the use of thin targets enabling the detection of low energetic recoils and thus the investigation of reactions at lower momentum transfer than before. Additionally, by utilizing the possibilities of beam cooling in storage rings the beam quality can be improved to the level of primary beams, thus allowing measurements almost free of background and contaminants.

The existing storage ring ESR at GSI, together with its internal gas-jet target, provides a unique opportunity to perform this kind of experiments on a smaller scale at the present. In autumn 2012, we successfully performed an experiment (E105) with stable ${ }^{58} \mathrm{Ni}$, as well as, radioactive ${ }^{56} \mathrm{Ni}$ beams interacting with $\mathrm{H}_{2}$ and ${ }^{4} \mathrm{He}$ targets. Besides the commissioning of our detection system we performed a physics program aiming at the measurement of the nuclear matter distribution of the doubly-magic ${ }^{56} \mathrm{Ni}$ by employing elastic proton scattering. The measured differential cross section will be analysed within the frame of the Glauber multiple-scattering theory. By using phenomenological density distributions, and by varying their free parameters, the calculated cross section will be fitted to the experimental data. In this way, the nuclear matter radius and radial matter distribution will be extracted (see for example [4] and references therein).

\section{Experimental Setup}

In the last few years, we have developed a UHV compatible detector setup mainly based on DSSDs for the target recoils [5]. To cope with the demanding vacuum requirements in a storage ring, the DSSDs act as active windows separating the UHV from an auxiliary vacuum where non-bakeable components can be placed. In this way, additional dead layers are avoided and a low energy threshold is maintained. With this vacuum solution, we have reached a pressure of $2 \times 10^{-10} \mathrm{mbar}$ in the target region of the ESR and $7 \times 10^{-8}$ mbar in the auxiliary vacuum. Figure 1 shows a schematic drawing of the prototype-detector setup around the gas-jet target of the ESR. The first detection system at a position close to $90^{\circ}$ is utilized for the investigation of elastic and inelastic scattering processes where, in inverse kinematics, a large dynamical range of energies need to be covered, i.e. starting from zero energy at $90^{\circ}$ laboratory scattering angle up to energies in the order of several tens of $\mathrm{MeV}$ (for example $65 \mathrm{MeV}$ already at $75^{\circ}$ laboratory scattering angle for ${ }^{56} \mathrm{Ni}(\mathrm{p}, \mathrm{p})^{56} \mathrm{Ni}$ at $400 \mathrm{MeV} / \mathrm{u}$ ). To cope with this dynamical range, the system is set up as a telescope consisting of one DSSD, which has an active area of $(6 \times 6) \mathrm{cm}^{2}$ divided into 128 strips at the front side and 64 orthogonal strips at the rear side, and two $6.5 \mathrm{~mm}$ thick $\mathrm{Si}(\mathrm{Li})$ detectors, which are segmented into $2 \times 4$ pads of $(2 \times 2) \mathrm{cm}^{2}$ each. They were placed in the auxiliary vacuum behind the DSSD, and were actively cooled during both the bake-out of the ESR and the experiment. 


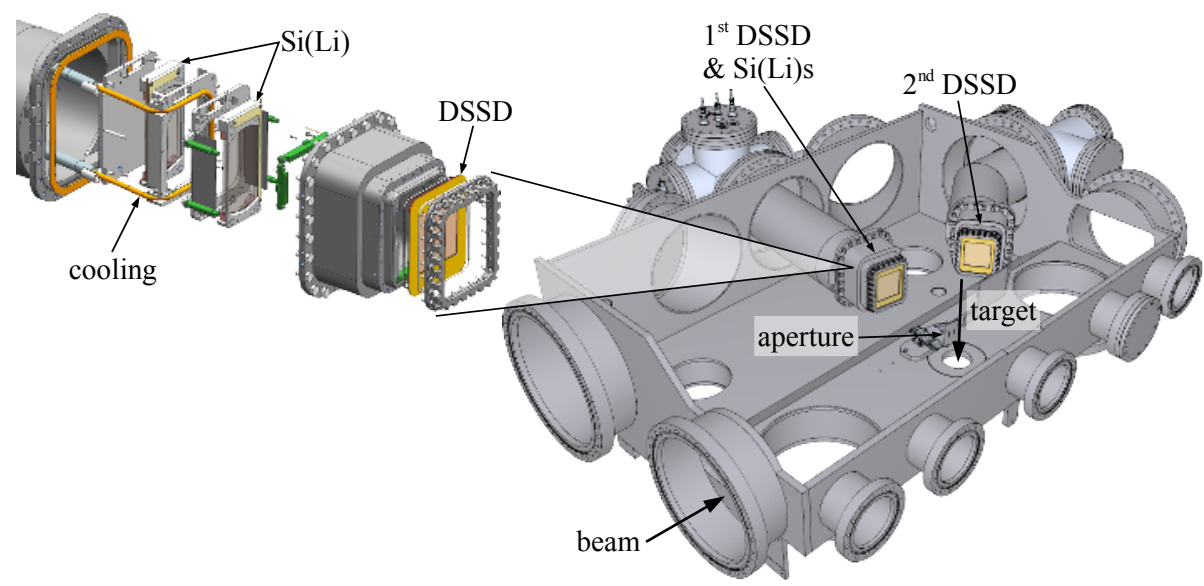

Figure 1. Schematic drawing of the EXL prototype-detector at the ESR.

Since the beam and the gas-jet target have extensions of approximately $3 \mathrm{~mm}$ (FWHM) and $5 \mathrm{~mm}$ (FWHM) respectively, it would not be possible to separate the kinematical bands for elastic and inelastic scattering. To reach the necessary angular resolution, a remotely moveable aperture with two piezo drives operated inside the UHV was placed in front of the target.

A second, single DSSD placed at forward angles is foreseen to study different reaction channels. Not shown in Figure 1 is a detection system for the projectile-like heavy ions further downstream of the target. It features an array of six silicon PIN-diodes directly placed in the UHV. Furthermore, a SSD was used in a vacuum pocket for the same purpose. To investigate the conditions for the detection of neutrons and gammas, we utilized ELENS (European Low Energy Neutron Spectrometer) [6] and a CsI detector placed outside the vacuum chamber.

\section{Preliminary Results}

As already outlined, the main physics goal of the experiment was the measurement of the nuclear matter distribution in ${ }^{56} \mathrm{Ni}$ by investigating ${ }^{56} \mathrm{Ni}(\mathrm{p}, \mathrm{p}){ }^{56} \mathrm{Ni}$ at an energy of $400 \mathrm{MeV} / \mathrm{u}$. By stacking the secondary beam coming from the FRS (FRagment Seperator), it was possible to achieve $3 \times 10^{6}$ ions stored in the ring. Together with the target density of approximately $2 \times 10^{13} \mathrm{~cm}^{-2} \mathrm{H}_{2}$ and the revolution frequency in the ESR of about $2 \mathrm{MHz}$ this yields a luminosity of about $10^{26}$ particles s $\mathrm{s}^{-1} \mathrm{~cm}^{-2}$ which was monitored during the experiment by constantly measuring both the beam current and the target density.

Figure 2 shows 2-dimensional plots of the reconstructed energy of the recoiling protons measured by the detector telescope close to $90^{\circ}$ as a function of detector strip-number defining the laboratory scattering angle. The data on the left side of Figure 2 were taken without using the slit aperture. By introducing the slit aperture and thus improving the angular resolution, the kinematical bands for both elastic scattering and inelastic scattering to the first $2^{+}$-state at $2.7 \mathrm{MeV}$ become nicely separated (Figure 2, right side). In both spectra a first minimum of the cross section can be seen at an energy of about $17 \mathrm{MeV}$. It should be noted here that the irregularity, which is emerging from the band of elastic scattering at around $6 \mathrm{MeV}$, arises from imperfect energy reconstruction in the detector telescope.

With the improved angular resolution it is now possible to gate on the elastically scattered events only, and thus deduce a cross section. In a next step, corrections for the losses of luminosity, which are introduced by the slit aperture, have to be applied. These corrections will be estimated with the help of GEANT4 simulations taking into account the realistic geometry of the setup. 


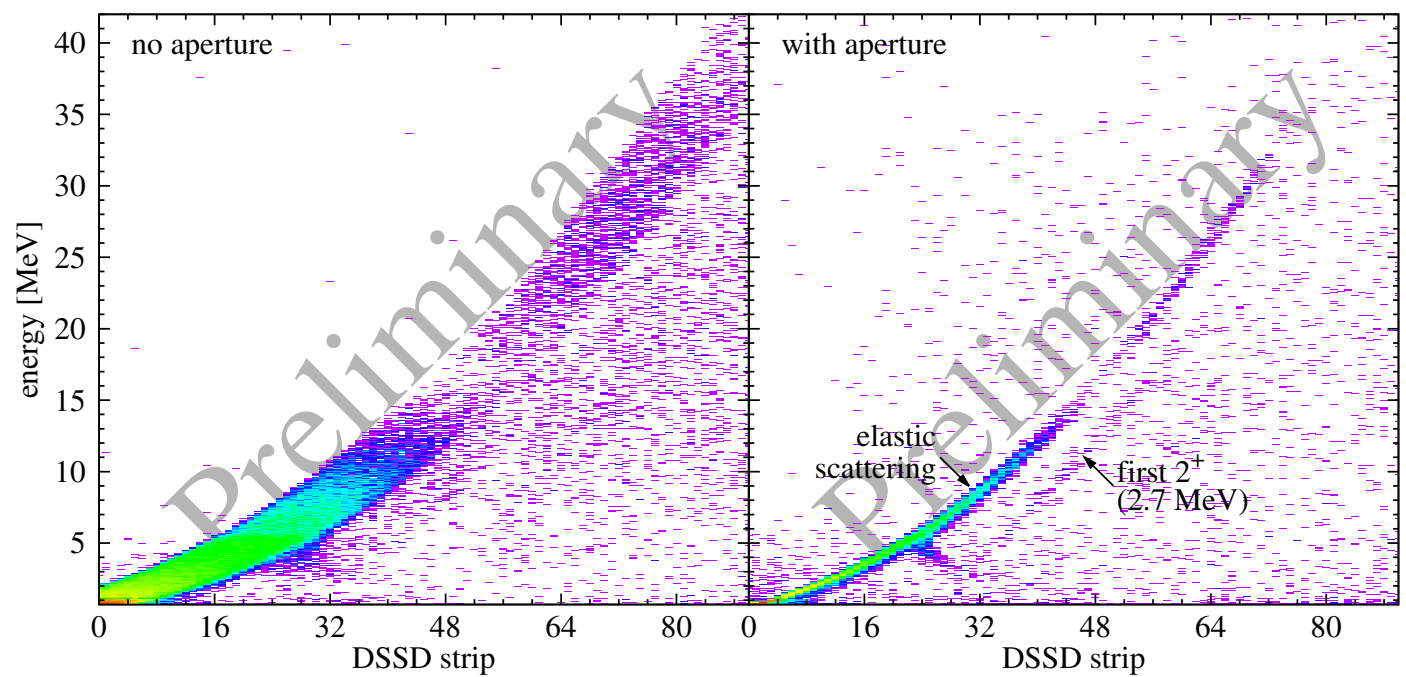

Figure 2. Reconstructed energy of recoiling protons vs. DSSD strip-number for ${ }^{56} \mathrm{Ni}(\mathrm{p}, \mathrm{p})^{56} \mathrm{Ni}$ and ${ }^{56} \mathrm{Ni}\left(\mathrm{p}, \mathrm{p}^{\prime}\right)^{56} \mathrm{Ni}^{\star}$; (left) without slit aperture; (right) with $1 \mathrm{~mm}$ slit aperture at $3 \mathrm{~cm}$ distance to the target in front of the $90^{\circ}$ telescope.

\section{Conclusion \& Outlook}

The experiment described here was the first of its kind and represents an essential milestone towards the realization of the EXL project at FAIR. By using the DSSDs as active windows, we were able to operate our detectors directly in the UHV without compromising a low energy threshold. A good angular resolution for the detector telescope located close to $90^{\circ}$ was maintained by using a slit aperture in front of the target. In the future, this will become obsolete thanks to reductions in the spatial extent of a new droplet target replacing the present gas-jet target, enabling interaction lengths much smaller than $1 \mathrm{~mm}$ [7]. The data analysis is currently in progress but already the preliminary results are very promising and demonstrate the feasibility of the intended program of EXL. Differential cross sections as a function of momentum transfer are in progress and, with the help of theoretical models, will yield the nuclear matter distribution of ${ }^{56} \mathrm{Ni}$.

After this successful experiment, an upgraded detector setup covering a substantially larger solid angle is envisaged to be implemented for further reaction experiments at the ESR.

This work was supported by BMBF (06DA9040I and 05P12RDFN8), the European Commission within the Seventh Framework Programme through IA-ENSAR (contract no. RII3-CT-2010-262010), HIC for FAIR and TU Darmstadt-GSI cooperation contract.

\section{References}

[1] http://www.fair-center.eu/for-users/experiments/nustar.html

[2] http://www.fair-center.eu

[3] H.H. Gutbrod et al. (Eds.), FAIR Baseline Technical Report, ISBN-3-9811298-0-6, Nov. 2006

[4] A.V. Dobrovolsky et al., Nuclear Physics A 766, 1 (2006)

[5] B. Streicher et al., Nucl. Instr. And Meth. A 654, 604 (2011)

[6] C. Langer et al., Nucl. Instr. And Meth. A 659, 411 (2011)

[7] M. Kühnel et al., Nucl. Instr. and Meth. A, 602, 411 (2009) 